\title{
ESTADO POBLACIONAL Y DISTRIBUCIÓN DEL COCODRILO DE PANTANO (CROCODYLUS MORELETII) EN REGIONES PRIORITARIAS DE PETÉN, GUATEMALA
}

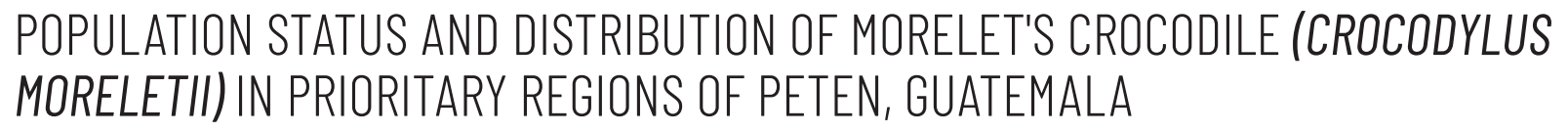

\author{
Valerie Andrea Corado García ${ }^{1 *}$, José Octavio Cajas Castillo² \& Javier Arturo Benítez-Moreno ${ }^{3,4}$ \\ ${ }^{1}$ Universidad del Valle de Guatemala, 18av. 11-95 Z.15, V.H.III, Guatemala. 01015. \\ ${ }^{2}$ Taxa, 45 Av. "A", 4-09 col. Tesoro BANVI, Z.2 de Mixco, Guatemala. \\ ${ }^{3}$ El Colegio de la Frontera Sur, Av. Centenario Km 5.5, 77014 Chetumal, Quintana Roo, México. \\ ${ }^{4}$ Cocodrilos de México, Matamoros y Bravo \#89, 95870 Catemaco, Veracruz, México. \\ *Correspondence: valeriegarci@gmail.com
}

Abstract.- The main purpose of the study was to provide information about the populations of Crocodylus moreletii in the Petén Department, Guatemala. The samplings were carried out during the rainy season of 2013 in 10 water bodies, categorized into open aquatic systems (rivers) and closed aquatic systems (lakes and lagoons). A total of 301 individuals were sighted, with an average Encounter Rate (ER) of 1.11 crocodiles $/ \mathrm{km}$ in open aquatic systems and 0.7 crocodiles $/ \mathrm{km}$ in closed aquatic systems. However, the ER of C. moreletii in Guatemala is low in comparison to Mexico and Belize, likely because of strong anthropogenic threats. Therefore, continuous monitoring of wild populations is recommended, as well as a community outreach program with human populations in the area to promote the conservation of the species as well as coexistence between crocodiles and communities.

Keywords.- Closed aquatic systems, crocodile conservation, encounter rate, open aquatic systems.

Resumen.- El objetivo del presente estudio fue brindar información sobre las poblaciones de Crocodylus moreletii en el departamento de Petén, Guatemala. Los muestreos se realizaron durante la época lluviosa del año 2013 en 10 cuerpos de agua, categorizados en sistemas acuáticos abiertos (ríos) y sistemas acuáticos cerrados (lagos y lagunas). Se avistaron un total de 301 individuos, con una Tasa de Encuentro promedio (TE) de 1.11 cocodrilos $/ \mathrm{km}$ en sistemas acuáticos abiertos y 0.7 cocodrilos $/ \mathrm{km}$ en sistemas acuáticos cerrados. Sin embargo, las TE de C. moreletii en Guatemala son bajas en comparación con las de México y Belice, probablemente como consecuencia de las fuertes amenazas antropogénicas. Por esto se recomienda un monitoreo continuo de las poblaciones silvestres, así como un programa de trabajo comunitario con las poblaciones humanas de la zona, con el propósito de promover la conservación de la especie desde la correcta coexistencia entre cocodrilos y humanos.

Palabras clave.- Conservación de cocodrilos, sistemas acuáticos abiertos, sistemas acuáticos cerrados, tasa de encuentro.

\section{INTRODUCCIÓN}

El cocodrilo de pantano (Crocodylus moreletii) es una especie cuyo ámbito de distribución se extiende desde Tamaulipas y el Golfo de México hasta la parte norte de Guatemala y Belice (Ross, 1987). En Guatemala, su ámbito se restringe específicamente en los departamentos de Petén, Izabal, Baja y Alta Verapaz (Köhler, 2003; Sánchez et al., 2011).

Los cocodrilos son considerados una especie clave en su ecosistema, al modificar la estructura y dinámica de su entorno, mediante la formación de pequeñas pozas y excavación de 
túneles o agujeros que sirven para almacenar agua dulce, influyendo en la composición vegetal promoviendo una mayor diversidad durante la época de sequía (Sánchez et al., 2011; Palmer \& Mazzotti, 2004). Además, es depredador clave en la regulación de la abundacia y comportamiento de depredadores de orden inferior, incluso pueden ser buenos indicadores de pesca (Somaweera et al., 2020). La importancia del cocodrilo radica también en la perspectiva antropológica, por formar parte de la cosmovisión de diversos grupos mayas, al ser tomado como un ser divino de alta jerarquía, encontrándose en tres niveles del cosmos: la tierra, el cielo y el inframundo (Arias, 2007). Este pensamiento resultó de la relación del cocodrilo con los espacios que lo rodean (e.g. cuevas, ríos, pantanos y lagunas) categorizándolo como un animal divino, incluso los mayas tenían la creencia que los "chillidos" de las crías anunciaban las lluvias (Arias, 2007).

En Guatemala, durante los años sesenta y setentas era común ver ejemplares de $C$. moreletii en la mayoría de los cuerpos de agua en Petén, como: el lago Petén Itzá, laguneta Petenchel y laguna Yaxhá; sin embargo, estas poblaciones silvestres han disminuido a causa de las grandes migraciones humanas (Campbell, 1998). Posteriormente, Lara (1990) estimó la estructura y tamaño poblacional de $C$. moreletii del lago Petén Itzá y las lagunas Petenchel, Sal Petén y Yahxá, reportando densidades de 1.95 cocodrilos $/ \mathrm{km}, 3.9$ cocodrilos $/ \mathrm{km}, 2.6$ cocodrilos $/ \mathrm{km}$ y 1.94 cocodrilos $/ \mathrm{km}$ respectivamente, con una estructura etaria conformada principalmente por subadultos, seguido de juveniles y adultos.

Castañeda (1998) estimó la densidad poblacional en el río Sacluc y San Pedro en época de secas, encontrando 4.35 cocodrilos $/ \mathrm{km}$ y 2.10 cocodrilos $/ \mathrm{km}$ respectivamente. También, reportó en el río Sacluc que los adultos estaban mayormente asociados a la vegetación riparia, mientras que juveniles y subadultos se asociaban a zonas con menor cobertura vegetal, a diferencia de lo que encontró en el río San Pedro, donde los juveniles se asociaban más a vegetación riparia y subadultos y adultos a zonas con menor cobertura vegetal. Finalmente, Castañeda et al. (2000) estimaron la densidad poblacional en los ríos San Pedro, Sacluc, Escondido, las lagunas Flor de Luna, Vista Hermosa, El Tintal, La Pista, Guayacán, laguneta El Perú y aguadas a orilla de la carretera Xan-Flor de Luna ubicadas en Petén, registrando una densidad promedio de todos los sitios de 4.3 cocodrilos/ $\mathrm{km}$ en $87 \mathrm{~km}$ recorridos. Además, los cocodrilos adultos presentaban mayor abundancia en vegetación riparia y carrizal, mientras que las crías y juveniles se asociaban más a vegetación de tipo carrizal.
Los estudios sobre la abundancia poblacional y estructura etaria de los crocodilianos son clave para conocer sitios prioritarios para su conservación, el uso específico del hábitat para reproducción y anidación, e identificar potenciales amenazas para la especie (Read et al., 2004; Aguilar-Olguín et al., 2020). Este tipo de información permite tomar decisiones en cuanto al manejo sostenible de la especie y el desarrollo de programas de conservación para mitigar las interacciones negativas entre cocodrilos-humanos (Tellez et al., 2016).

En este estudio se presenta información sobre la abundancia relativa de las poblaciones silvestres de Crocodylus moreletii, y su asociación entre la estructura etaria y la vegetación en los distintos cuerpos de agua del departamento de Petén, Guatemala.

\section{MATERIALES Y MÉTODOS}

\section{Descripción de los sitios de muestreo}

El estudio se llevó a cabo en distintos cuerpos de agua en Petén, Guatemala. Los sitios fueron seleccionaron en función de las áreas priorizadas por Sánchez et al., 2011: a) río San PedroLaguna del Tigre: río San Pedro (RSP), río Sacluc (RS) y laguneta El Perú (LP); b) río Usumacinta (RU); c) río La Pasión-Petexbatún: río La Pasión (RLP), río Petexbatún y laguna Petexbatún (RyLP); y d) la laguna Zona Central de Petén: lago Petén Itzá (LPI), laguna Yahxá (LY) y laguna Sacnab (LS). Los cuerpos de agua monitoreados se clasificaron en sistemas acuáticos abiertos (ríos) y sistemas acuáticos cerrados (lagunas) (Fig. 1).

De los diez sitios seleccionados para esta investigación, ocho se encuentran dentro de la Reserva de la Biosfera Maya (RBM), y los otros dos al suroeste de Petén, área que no cuenta con planes de conservación. La RBM posee una extensión de 2,090,667 ha, con clima tropical cálido-húmedo y una temperatura promedio que oscila entre $20^{\circ} \mathrm{C}$ para la mínima y $33^{\circ} \mathrm{C}$ para la máxima (CONAP, 2015).

La Reserva está conformada por tres zonas principales: Zona de Amortiguamiento (cubre el $23 \%$ de la reserva), Zona de Usos Múltiples (38\% cubre de la reserva) y Zona Núcleo (cubre el 39\% de la reserva). Dentro de las comunidades vegetales relevantes que posee la RBM, se encuentran el bosque ripario y un remanente de manglar (Rhyzophora mangle); siendo de suma importancia en la conservación, debido a la diversidad de flora y fauna que alberga, especialmente dentro del Parque Nacional Laguna del Tigre (PNLT) (CONAP, 2015). 


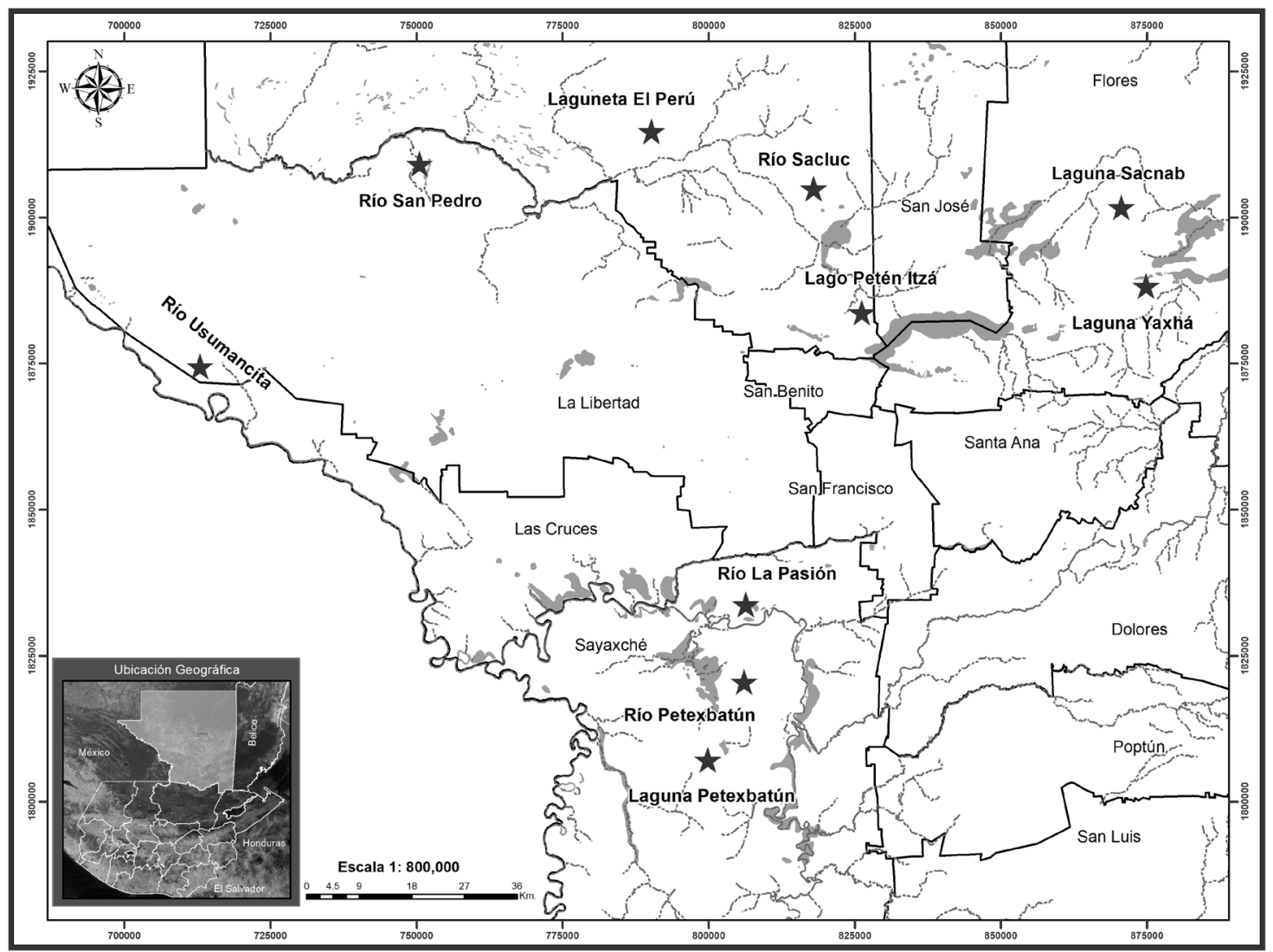

Figure 1. Sampling sites for Crocodylus moreletii in Petén, Guatemala (black stars).

Figura 1. Sitios de muestreo para Crocodylus moreletii en Petén, Guatemala (estrellas negras).

El río La Pasión y río-laguna Petexbatún se encuentran dentro del municipio de Sayaxché, al suroeste del departamento de Petén. Estos cuerpos acuáticos se localizan fuera de la RBM, lo que ha condicionado la protección de los recursos naturales del área; dentro de las principales actividades pecuarias se encuentran la agricultura de corte y quema, fincas de ganado, tala, plantaciones de palma, entre otras, que pueden estar generando un alto impacto en la dinámica natural de la zona. El recurso hídrico de ambos ríos está siendo altamente contaminado por hidrocarburos y aguas residuales, las cuales no cuentan con un sistema de tratamiento (CONAP, 2008).

\section{Características del muestreo}

Los sitios de estudio fueron visitados una vez durante la época de lluvias entre julio y octubre de 2013. Los monitoreos se realizaron a bordo de una lancha de fibra de vidrio de $7.3 \mathrm{~m}$ de longitud y con un motor de 70 caballos de fuerza, a una velocidad constante de $5 \mathrm{~km} / \mathrm{h}$ (Sánchez et al., 2011). La distancia total recorrida en los sistemas acuáticos abiertos y cerrados fue de $220.86 \mathrm{~km} \mathrm{y}$ $171.69 \mathrm{~km}$, respectivamente.

La caracterización del tipo de vegetación y la clasificación de las estructuras etarias se establecieron de acuerdo con el Manual Trinacional de Monitoreo del Cocodrilo de Pantano (Sánchez et al., 2011) para estandarizar la toma de datos a nivel regional entre México, Belice y Guatemala. De esta manera, la vegetación marginal de los cuerpos de agua se categorizó en: carrizal, hábitat boscoso, pastizal, tular, lirial y nenufaral (Sánchez et al., 2011). Para estimar la abundancia relativa se empleó el método de Detección Visual Nocturna (DVN), clasificando a los individuos avistados en: crías (cocodrilo que ha superado el primer invierno; menores a $0.5 \mathrm{~m})$, juveniles $(0.51-1.0 \mathrm{~m})$, sub-adultos (1.01 -1.5 m), adultos (1.51m a 2) y adultos grandes (de $2.01 \mathrm{mo}$ más). Cuando no fue posible la asignación categórica por talla, el avistamiento fue registrado como "solo ojos" (Sánchez et al., 2011). Adicionalmente cada avistamiento fue georeferenciado 
utilizando un Sistema de Posicionamiento Global (GPSMap 62 Garmin) en coordenadas geográficas.

\section{Análisis estadístico}

La abundancia relativa se estimó con base en la tasa de encuentro (TE), la cual se calcula como el número de cocodrilos avistados entre la distancia recorrida en km lineales (Sánchez et al., 2011). Para determinar la estructura etaria a lo largo de los meses estudiados, se utilizó el número de individuos de cada categoría etaria entre el total de individuos registrados mensualmente en cada muestreo (Fig. 2). Se empleó la prueba de Kruskal-Wallis para determinar si existe diferencia significativa entre los diez sitios de muestreo en las estructuras etarias de las poblaciones de Crocodylus moreletii.

Por último, se utilizó el Coeficiente de correlación de Spearman para determinar si existe asociación entre algún tipo de vegetación y las categorías etarias en las de los cocodrilos avistados. Los análisis se realizaron a través del software estadístico PAST v. 2.17 y se aplicó un nivel de significancia de P $\leq 0.05$ (Hammer et al., 2013).

\section{RESULTADOS}

Se registraron un total de 301 individuos de C. moreletii en 392.55 $\mathrm{km}$ recorridos. De los cuales 226 se observaron a lo largo de $220.86 \mathrm{~km}$ en los sistemas acuáticos abiertos, y 75 individuos a lo largo de $171.69 \mathrm{~km}$ en los sistemas acuáticos cerrados.

Los sistemas acuáticos abiertos presentaron una TE promedio de 1.11 cocodrilos/kilómetro, siendo en el río Sacluc donde se registró el mayor número de avistamientos ( $\mathrm{TE}=2.41$ cocodrilos/ $\mathrm{km}$ ) y en el río La Pasión la menor tasa de avistamientos (TE = 0.05 cocodrilos $/ \mathrm{km}$ ) (Tabla 1$)$. Mientras que, en los sistemas acuáticos cerrados se registró una menor TE ( 0.70 cocodrilos/ $\mathrm{km})$, encontrando en la laguna Yaxhá la TE más alta (2.14 cocodrilos $/ \mathrm{km}$ ), y en la laguna Petexbatún no se observó ningún cocodrilo (Tabla 1).

En cuanto a la estructura etaria, las proporciones de cada categoría variaron a través de los meses de estudio y de los sitios. El río Usumacinta presentó la mayor proporción de crías, la laguna Yaxhá mostró la mayor proporción de jóvenes, y el río San Pedro manifestó la mayor cantidad de sub-adultos, adultos y adultos grandes, siendo este el sitio más diverso en tallas

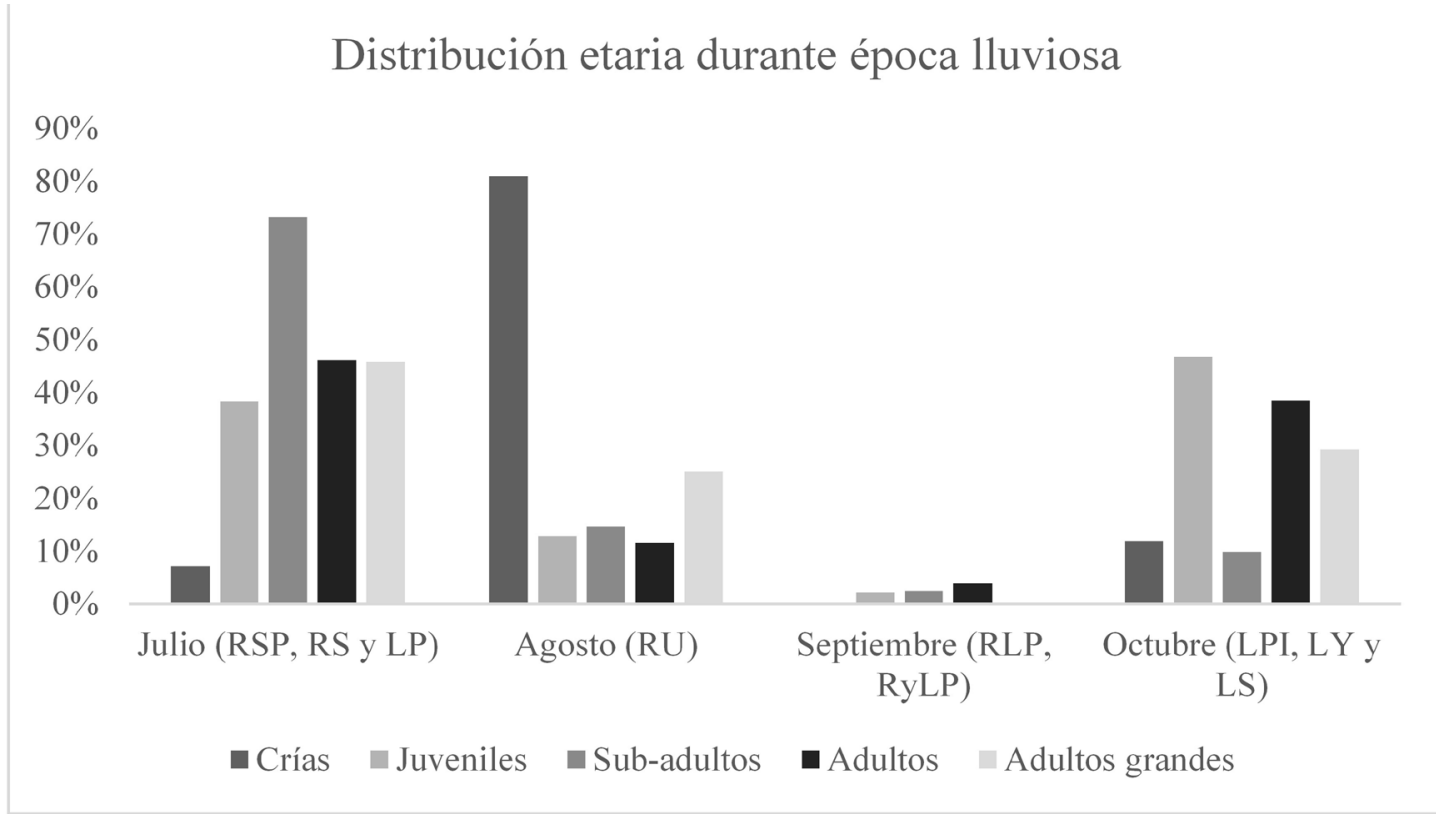

Figure 2. Age distribution of Crocodylus moreletii during the rainy season in the ten sampled sites in Petén, Guatemala. Key: *RSP (San Pedro river), RS (Sacluc river), LP (El Perú lagoon), UR (Usumacinta river), RLP (La Pasión river), RyLP (river and lagoon Petexbatún), LPI (lake Petén Itzá), LY (Yaxhá lagoon) and LS (Sacnab lagoon).

Figura 2. Distribución etaria de Crocodylus moreletii durante la época lluviosa en los diez sitios muestreados de Petén, Guatemala. Clave: *RSP (río San Pedro), RS (río Sacluc), LP (laguneta El Perú), RU (río Usumacinta), RLP (río La Pasión), RyLP (río y laguna Petexbatún), LPI (lago Petén Itzá), LY (laguna Yaxhá) y LS (laguna Sacnab). 
Table 1. Encounter rate of Crocodylus moreletii in open and closed aquatic systems in Petén, Guatemala during the rainy season in 2013.

Tabla 1. Tasa de encuentro de Crocodylus moreletii en sistemas acuáticos abiertos y cerrados en Petén, Guatemala durante la época lluviosa en 2013.

\section{Sistema ACUÁtico ABIERTO}

\begin{tabular}{lccc}
\multicolumn{1}{c}{ Sitios } & $\begin{array}{c}\text { Km } \\
\text { recorridos }\end{array}$ & \# individuos & $\begin{array}{c}\text { Tasa de } \\
\text { encuentro } \\
\text { (TE) }\end{array}$ \\
\hline Rio Sacluc & 8.7 & 21 & 2.41 \\
Río San Pedro & 57.5 & 120 & 2.09 \\
Río Usumacinta & 96.18 & 80 & 0.83 \\
Río Petexbatún & 18.18 & 3 & 0.17 \\
Río La Pasión & 40.3 & 2 & 0.05 \\
Total & $\mathbf{2 2 0 . 8 6}$ & $\mathbf{2 2 6}$ & $\mathbf{N} / \mathbf{A}$ \\
Promedio de TE & - & - & 1.11 \\
Desviación estándar TE & - & - & 1.09 \\
\hline
\end{tabular}

\section{Sistema ACuÁtico ABIERTO}

\begin{tabular}{lccc}
\multicolumn{1}{c}{ Sitios } & $\begin{array}{c}\text { Km } \\
\text { recorridos }\end{array}$ & \# individuos & $\begin{array}{c}\text { Tasa de } \\
\text { encuentro } \\
\text { (TE) }\end{array}$ \\
\hline Laguneta el Perú & 21.3 & 9 & 0.42 \\
Lago Petén Itzá & 95.51 & 7 & 0.07 \\
Laguna Sacnab & 12.3 & 11 & 0.89 \\
Laguna Yaxhá & 22.4 & 48 & 2.14 \\
Laguna Petexbatún & 20.18 & 0 & 0 \\
Total & $\mathbf{1 7 1 . 6 9}$ & $\mathbf{7 5}$ & $\mathbf{N} / \mathbf{A}$ \\
Promedio de TE & - & - & 0.7 \\
Desviación estándar TE & - & - & 0.88 \\
\hline
\end{tabular}

(Tabla 2).

Se encontró diferencia estadísticamente significativa entre los sitios de muestreo para los diferentes grupos etarios de las poblaciones de $C$. moreletii $(H=31.56, p=0.0002$ ). Siendo los ríos San Pedro, Usumacinta y laguna Yaxhá los sitios que presentan diferencias respecto al resto de los cuerpos de agua (Tabla 3).
Las correlaciones de Spearman indican que los hábitats boscosos y carrizal están asociados a las categorías etarias menores (crías, juvenily subadulto), mientras que tular y pastizal se asocia a las categorías mayores (adulto y adulto grande); sin embargo, ninguna de estas fue estadísticamente significativa. Por último, la vegetación de tipo lirial y nenufaral muestran una correlación negativa con subadultos, adultos y adultos grandes (Tabla 4).

\section{DISCUSIÓN Y CONCLUSIONES}

La Tasa de Encuentro promedio de C. moreletii en los sistemas cerrados fuemenor $(\overline{\mathrm{x}}=0.7)$ que elencontrado en sistemas abiertos $(\overline{\mathrm{X}}=1.1)$. Estas diferencias se pueden deber, principalmente, a la época de muestreo (época lluviosa) donde los niveles de agua incrementan formando canales intermitentes, especialmente en lagunas, permitiendo a los cocodrilos desplazarse hacia otros sitios (Dever et al., 2002; Cedeño-Vásquez \& Pérez-Rivera, 2010; Rodas-Trejo et al., 2017).

Las TE obtenidas en este estudio para los ríos Sacluc y San Pedro (2.41 y 2.09 cocodrilos $/ \mathrm{km}$, respectivamente), fueron similares a las reportadas por Castañeda (1998) y Castañeda et al. (2000), quien registró 4.35 y 2.10 cocodrilos $/ \mathrm{km}$, mientras que, en el 2000 , los autores reportaron valores de 2.00 y 0.61 cocodrilos/ $\mathrm{km}$ para los mismos sitios, respectivamente. Adicionalmente, en México Cedeño-Vásquez et al. (2006) en río Hondo, Quintana Roo registraron una TE promedio de 3 cocodrilos $/ \mathrm{km}$ con muestreos en época seca y lluviosa; mientras que Villalobos-Zapata y Mendoza Vega (2010) registraron una TE de 15.53 cocodrilos/ $\mathrm{km}$ en el río Verde de Hampolol, de la Reserva de la Biosfera de los Petenes, también en ambas estaciones. En el caso de Belice, Platt \& Thorbjarnarson (2000) realizaron muestreos de varios ríos y riachuelos entre 1992 y 1997 en el norte del país, reportando una TE promedio de 0.95 cocodrilos $/ \mathrm{km}$. Asimismo, Tellez et al. (2017), reportaron una TE promedio de 3.6 cocodrilos $/ \mathrm{km}$ para los ríos Raspaculo y Macal con muestreos en abril (época seca) y junio (época lluviosa).

Con base en las TE reportadas a nivel regional, se puede inferir que las poblaciones silvestres de Guatemala son bajas. No obstante, la escasez de estudios sobre cocodrilos en Guatemala limita concluir que las poblaciones silvestres sean o no estables. Los únicos sitios que permiten hacer una comparación a lo largo de los años son los ríos San Pedro y Sacluc, cuyas poblaciones silvestres han mostrado estabilidad temporal de acuerdo con los estudios previamente mencionados; pudiéndose clasificar como áreas importantes para la conservación de C. moreletii. 
Table 2. Age distribution of Crocodylus moreletii at the sampling sites in Petén, Guatemala during the rainy season in 2013.

Tabla 2. Distribución etaria de Crocodylus moreletii en los sitios de muestreo en Petén, Guatemala durante la época lluviosa en 2013.

\begin{tabular}{lccccccc}
\hline \multicolumn{1}{c}{ Sitios } & Cría & Juvenil & Sub-Adulto & Adulto & Adulto Grande & Sólo ojos & Total \\
\hline Río Sacluc & 0 & 7 & 2 & 0 & 0 & 12 & 21 \\
Río San Pedro & 3 & 8 & 26 & 11 & 11 & 61 & 120 \\
Río Usumacinta & 34 & 6 & 6 & 3 & 6 & 25 & 80 \\
Río Petexbatún & 0 & 1 & 1 & 0 & 0 & 1 & 3 \\
Río La Pasión & 0 & 0 & 0 & 1 & 0 & 1 & 2 \\
Laguneta el Perú & 0 & 3 & 2 & 1 & 0 & 3 & 9 \\
Lago Petén Itzá & 1 & 4 & 0 & 1 & 0 & 1 & 7 \\
Laguna Sacnab & 0 & 0 & 0 & 3 & 5 & 3 & 11 \\
Laguna Yaxhá & 4 & 18 & 4 & 6 & 2 & 14 & 48 \\
Laguna Petexbatún & 0 & 0 & 0 & 0 & 0 & 0 & 0 \\
Total \# de individuos & $\mathbf{4 2}$ & $\mathbf{4 7}$ & $\mathbf{4 1}$ & $\mathbf{2 6}$ & $\mathbf{2 4}$ & $\mathbf{1 2 1}$ & $\mathbf{3 0 1}$ \\
\hline
\end{tabular}

Table 3. Krukal-Wallis analysis between sampling sites using age categories of Crocodylus moreletii in Peten, Guatemala during the rainy season in 2013. $p=0.0002371$. Key: *RSP (San Pedro river), RS (Sacluc river), LP (El Perú lagoon), UR (Usumacinta river), RLP (La Pasión river), RyLP (river and lagoon Petexbatún), LPI (lake Petén Itzá), LY (Yaxhá lagoon) and LS (Sacnab lagoon).

Tabla 3. Análisis de Kruskal-Wallis entre sitios muestreados utilizando categorías etarias de Crocodylus moreletii en Petén, Guatemala durante la época lluviosa en 2013 . p = 0.0002371. Clave: *RSP (río San Pedro), RS (río Sacluc), LP (laguneta El Perú), RU (río Usumacinta), RLP (río La Pasión), RyLP (río y laguna Petexbatún), LPI (lago Petén Itzá), LY (laguna Yaxhá) y LS (laguna Sacnab).

\begin{tabular}{|c|c|c|c|c|c|c|c|c|c|}
\hline Sitios & RSP & RS & LP & RU & RLP & $\mathrm{RP}$ & LP & LPI & LY \\
\hline \multicolumn{10}{|l|}{ RSP } \\
\hline RS & 0.01095 & & & & & & & & \\
\hline LP & 0.01371 & 0.93660 & & & & & & & \\
\hline $\mathrm{RU}$ & 0.77640 & 0.02380 & 0.02920 & & & & & & \\
\hline RLP & 0.00071 & 0.40060 & 0.35760 & 0.00193 & & & & & \\
\hline $\mathrm{RP}$ & 0.00216 & 0.60130 & 0.54720 & 0.00539 & 0.75050 & & & & \\
\hline LP & 0.00021 & 0.24660 & 0.21570 & 0.00063 & 0.75050 & 0.52470 & & & \\
\hline LPI & 0.01286 & 0.95470 & 0.98190 & 0.02756 & 0.36950 & 0.56240 & 0.22420 & & \\
\hline LY & 0.53960 & 0.05349 & 0.06411 & 0.74190 & 0.00560 & 0.01415 & 0.00201 & 0.06091 & \\
\hline LS & 0.00993 & 0.97280 & 0.90960 & 0.02177 & 0.42000 & 0.62530 & 0.26080 & 0.92760 & 0.04940 \\
\hline
\end{tabular}


Table 4. Spearman Correlation Matrix between vegetation types and age categories of Crocodylus moreletii at the sampling sites in Petén, Guatemala during the rainy season in 2013.

Tabla 4. Matriz de Correlación de Spearman entre tipos de vegetación y categorías etarias de Crocodylus moreletii en los sitios de muestreo en Petén, Guatemala durante la época lluviosa en 2013.

\begin{tabular}{|c|c|c|c|c|c|c|c|c|c|c|c|c|}
\hline \multirow[t]{2}{*}{ Talla } & \multicolumn{2}{|c|}{ Carrizal } & \multicolumn{2}{|c|}{ Hábitat boscoso } & \multicolumn{2}{|c|}{ Pastizal } & \multicolumn{2}{|c|}{ Tular } & \multicolumn{2}{|c|}{ Lirial } & \multicolumn{2}{|c|}{ Nenufaral } \\
\hline & $\mathrm{R}^{2}$ & $\mathrm{p}$ & $R^{2}$ & $p$ & $\mathrm{R}^{2}$ & $p$ & $\mathrm{R}^{2}$ & p & $\mathrm{R}^{2}$ & $p$ & $\mathrm{R}^{2}$ & $p$ \\
\hline Cría & 0.1444 & 0.2110 & 0.3086 & 0.3801 & -0.6251 & 0.2592 & 0.1772 & 0.1353 & 0.1961 & 0.4655 & 0.1961 & 0.4655 \\
\hline Juvenil & 0.3292 & 0.6496 & 0.3801 & 0.4987 & -0.6810 & 0.4199 & 0.0228 & 0.0379 & 0.0587 & 0.6263 & 0.0587 & 0.6263 \\
\hline Sub-adulto & 0.0841 & 0.7350 & 0.4714 & 0.6928 & -0.4630 & 0.8904 & -0.3140 & 0.1205 & -0.3604 & 0.8691 & -0.3604 & 0.8691 \\
\hline Adulto & 0.3891 & 0.3450 & -0.1234 & 0.5869 & -0.6134 & 0.0574 & 0.3929 & 0.0662 & -0.0597 & 0.2297 & -0.0597 & 0.2297 \\
\hline Adulto grande & 0.1162 & 0.4859 & 0.0797 & 0.6320 & -0.6251 & 0.2293 & 0.2152 & 0.1353 & -0.2615 & 0.4655 & -0.2615 & 0.4655 \\
\hline
\end{tabular}

El río La Pasión y río y laguna Petexbatún fueron los sitios con la TE más baja de los 10 cuerpos de agua estudiados (0.05 cocodrilos $/ \mathrm{km}, \quad 0.17$ cocodrilos $/ \mathrm{km}$ y 0 cocodrilos/ $\mathrm{km}$, respectivamente), lo cual puede deberse a un alto nivel de perturbación del ecosistema por la elevada presencia de actividades antropogénicas alrededor de estos sitios. En estos cuerpos de agua se ha reportado pesca con trasmallos, deforestación por avance de la frontera agrícola, registro de cacería ilegal, sobrepesca, contaminación por agroquímicos, entre otros factores de riesgo que perturban la abundancia de cocodrilos (CONAP, 2008). Estos factores pueden provocar un comportamiento evasivo de los cocodrilos dificultando el avistamiento durante los muestreos y una disminución de la población como consecuencia de estas actividades (Cruz-Paz et al., 2018).

El sistema acuático cerrado que obtuvo mayor TE fue la laguna Yaxhá (2.14 cocodrilos $/ \mathrm{km}$ ), seguido de la laguna Sacnab y laguneta El Perú ( 0.89 cocodrilos/km y 0.42 cocodrilos $/ \mathrm{km}$ ). Las lagunas Yaxhá y Sacnab están ubicadas dentro de un importante sitio arqueológico (Parque Nacional Yaxhá-Nakum-Naranjo) que cuenta con una eficiente vigilancia del área (CONAPDGPCN/MICUDE-, 2015). Aunque ambas lagunas están dentro del mismo parque, la variación en la abundancia de cocodrilos probablemente pudo deberse a un mayor número de refugios y recursos alimenticios en Yaxhá, principalmente por la alta diversidad de ictiofauna nativa y flora que alberga (hábitat boscoso, carrizal y tular) (Reyes Morales et al., 2009; CONAP, 2015).

Mientras que, Sacnab puede ser un sitio de importancia para el apareamiento por ser un cuerpo de agua provisto de hábitat boscoso y tular, lo cual se puede asociar a la alta presencia de cocodrilos adultos y adultos grandes que se registró (Reyes Morales et al., 2009; Villegas \& Reynoso, 2013) (Tabla 2).

En comparación con otros estudios, Castañeda (2000) reporta una TE promedio de 1 cocodrilo/km y 6 cocodrilos $/ \mathrm{km}$ en las lagunas Tintal y El Burro, Petén, Guatemala en época de lluvias; mientras que Castañeda et al. (2000) reportan una TE de 4.40 cocodrilos/ $\mathrm{km}$ en época de secas para la laguneta El Perú, ubicada dentro del Parque Nacional Laguna del Tigre, Petén. Sin embargo, para este estudio la TE ( 0.42 cocodrilos $/ \mathrm{km}$ ) obtenida para la laguneta El Perú fue mucho menor durante la época de lluvias; situación que pudo deberse a la migración de hembras hacia sitios que presentan condiciones favorables, así como: i) áreas para refugio de crías, y ii) una vegetación con escombros leñosos, hojas y tierra para la construcción de nidos (EscobedoGalván et al., 2011). Asimismo, Castañeda et al. (2000) reportan un alto porcentaje de adultos, lo cual puede influir en el comportamiento reproductivo de las hembras y otras especies de reptiles que tienen una tendencia similar (Barahona \& López, 2015) afectando la abundancia local durante la reproducción.

En relación con los estudios en México, Cedeño-Vásquez et al. (2006) y Cedeño-Vásquez \& Pérez-Rivera (2010), registraron una TE promedio de 7.57 cocodrilos/km en sistemas lagunares al Sureste de Quintana Roo y una TE máxima de 45.6 cocodrilos/ km para Laguna Esmeralda, Quintana Roo, respectivamente; ambos estudios se llevaron a cabo durante las épocas seca y lluviosa. Mientras que, Platt \& Thorbjarnarson (2000) registraron una TE promedio de 6.11 cocodrilos $/ \mathrm{km}$ en lagunas aluviales y 8.20 cocodrilos $/ \mathrm{km}$ en lagunas no aluviales al norte de Belice durante monitoreos en época seca y lluviosa entre 1992 y 
1997. Por consiguiente, las TE en las lagunas Petexbatún, Yaxhá, Sacnab y El Perú (2.14 cocodrilos/km, 0.89 cocodrilos $/ \mathrm{km}, 0.42$ cocodrilos $/ \mathrm{km}$ y o cocodrilos $/ \mathrm{km}$ ) son muy bajas en comparación con las registradas en México y Belice. Sin embargo, se observa el contraste de los cuerpos de agua que están dentro y fuera de áreas protegidas.

El mayor conteo de crías se realizó en el río Usumacinta (Tabla 2), presentando el pico más alto en el mes de agosto, lo cual puede tener relación con la época de eclosión de las crías (Castañeda, 1998; Castañeda et al., 2000). Asimismo, el río cuenta con una alta diversidad de fauna, especialmente de especies dulceacuícolas como insectos acuáticos, anfibios, crustáceos, entre otros, siendo estas la dieta principal para crías y juveniles (Platt et al., 2006; Amezcua et al., 2017; Castillo et al., 2018, Mendoza-Carranza et al., 2018) y una alta diversidad de flora adecuada para su supervivencia (Cedeño-Vásquez \& PérezRivera, 2010; Rueda-Cordero et al., 2017; Tellez et al., 2017). Sin embargo, la abundancia de las categorías de mayor talla en este cuerpo de agua fue más baja, probablemente porque el sitio está sujeto a altas tasas de deforestación, cacería ilegal de fauna, asentamientos humanos, avance de la frontera agrícola, entre otros (March \& Castro, 2010; Cruz-Paz et al., 2018). Posteriormente, se registró una disminución progresiva de crías en septiembre y octubre (Fig. 2) esto puede deberse a mortalidad natural, depredación y crecimiento (Cedeño-Vásquez \& PérezRivera, 2010; Cedeño-Vásquez, 2016).

Las correlaciones obtenidas sugieren que los cuerpos de agua cuyos márgenes han sido desprovistos de cobertura vegetal original tienen un efecto negativo sobre la presencia de cocodrilos, en particular para las crías, juveniles y subadultos (Tabla 4). Esta condición causa un aumento en la exposición a amenazas tanto antropogénicas como naturales, así como la depredación de adultos hacia tallas menores, mortalidad de los juveniles durante su transición a subadultos o desplazamiento de juveniles y subadultos por adultos en época de reproducción (Cedeño-Vásquez et al., 2006; Escobedo-Galván, 2008; CedeñoVásquez \& Pérez-Rivera, 2010).

El hábitat boscoso y carrizal fueron los tipos de vegetación que favorecieron a las crías, juveniles y subadultos (Tabla 4), probablemente porque brinda mayor protección y recursos alimenticios. Lo cual concuerda con lo dicho por Cedeño et al. (2006) y Villegas \& Reynoso (2013), que el hábitat boscoso es de suma importancia para el forrajeo y anidación, además los adultos tienen mayor preferencia por aguas abiertas (Tellez et al., 2017). Los adultos y adultos grandes presentaron mayor tendencia hacia pastizal y tular $(\mathrm{R} 2=0.61341$ y $\mathrm{R} 2=0.39291$ respectivamente), posiblemente porque lo utilizan para la construcción de nidos, sitios de refugio por la densa vegetación dificultando el acceso a humanos, entre otros (Castañeda et al., 2000; Villegas \& Reynoso, 2013). Aunque las correlaciones no son estadísticamente significativas, los valores obtenidos sugieren que los cocodrilos estarían evitando zonas desprovistas de vegetación y sitios de riesgo.

En conclusión, las poblaciones silvestres de Crocodylus moreletii en Petén, Guatemala se encuentran reducidas en comparación con las distribuidas en México y Belice. Asimismo, se observó la importancia de los hábitats boscosos y carrizales para la estructura poblacional de cocodrilos, tanto en sus etapas de vida más vulnerables como en la adultez por la protección y recursos alimenticios. Con lo discutido anteriormente, se podría inferir que hay una tendencia de mayor densidad de cocodrilos en cuerpos de agua cuya vegetación no ha sido modificada por impactos antropogénicos. Sin embargo, se requiere de un estudio más específico y con más réplicas para comparar la asociación de estos entre sitios conservados y no conservados.

Adicionalmente se recomienda mantener monitoreos anuales de C. moreletii tanto en época seca como lluviosa con el fin de evaluar la fluctuación de los grupos etarios a lo largo de los años. Se sugiere que, además de los estudios biológicos de la especie, se desarrollen proyectos comunitarios para mejorar las artes de pesca y reducir la cacería, mediante un cambio en la percepción y el conocimiento sobre la importancia y el valor intrínseco de los cocodrilos. Esto permitiría mitigar, en la medida de lo posible, los impactos de origen antropogénico en los cuerpos acuáticos y asegurar un manejo adecuado de conservación para obtener posteriormente un beneficio económico de manera sustentable.

Agradecimientos.- Se agradece al Fondo Nacional para la Conservación (FONACON) por financiar este proyecto con número de identificación F16/2013, al Consejo Nacional de Áreas Protegidas (CONAP) por otorgar la Licencia de Investigación No. 008/2014 con No. Reg. I-001-2011 y el apoyo que brindaron los técnicos de campo para la ejecución de esta. Además, al M.Sc. Luís Estuardo Ríos González, director de la Maestría en Gestión Ambiental y Sostenibilidad de la Universidad del Valle de Guatemala (UVG), por el acompañamiento y apoyo académico.

\section{LITERATURA CITADA}

Aguilar-Olguín, S., M. Cruz Rivera-Rodríguez, H. HernándezHurtado, R. González-Trujillo \& M.M. Ramírez-Martínez. 2020. 
Effect of vegetation and abiotic factors on the abundance and population structure of Crocodylus acutus (Cuvier, 1806) in coastal lagoons of Colima. Amphibian and Reptile Conservation. 14:174182.

Amezcua, I., G. Carreón, J. Márquez, R.M. Vidal, I. Burgués, S. Cordero \& J. Reid. 2007. Tenosique: análisis económicoambiental de un proyecto hidroeléctrico en el río Usumacinta. Conservation Strategy Fund, Serie Técnica No. 10.

Arias T.E. 2007. El cocodrilo en la Región Maya Yokot'an. Un acercamiento antropológico a la actualidad del Ambiente en Tabasco. Itinerarios 6:101-122.

Barahona, R. \& J. López. 2015. Estructura poblacional de la tortuga blanca, Dermatemys mawii Gray (Testudines, Dermatemydidae), en la Laguna El Perú, Petén, Guatemala. Revista Científica de la Facultad de Ciencias Químicas y Farmacia 25:75-86.

Castañeda, F. 1998. Situación actual y propuesta de plan de manejo para Crocodylus moreletii (Bibrón \& Dúmeril, 1851) (Reptilia: Crocodylidae), en el área de influencia de la Estación Biológica "Las Guacamayas", Parque Nacional Laguna del Tigre, Departamento de Petén, Guatemala. Tesis de Licenciatura. Facultad de Ciencias Químicas y Farmacia, Universidad de San Carlos de Guatemala. Guatemala.

Castañeda, F. 2000. Estudio poblacional de Crocodylus moreletii en las lagunetas El Tintal y El Burro. Informe Final para la Finca Santuarios Ecológicos “El Retiro", Macanche, Petén. Guatemala.

Castañeda, F., O. Lara \& A. Queral-Regil. 2000. The herpetofauna of Laguna del Tigre National Park, Peten, Guatemala, with an emphasis on populations of the Morelet's crocodile (Crocodylus moreletii). Pp. 61-66. En Bestelmeyer, Brandon \& E. Leeane (Eds.). Evaluación biológica de los sistemas acuáticos del Parque Nacional Laguna del Tigre, Petén, Guatemala. Boletín RAP de evaluación biológica 16, Conservation International. Washington, D.C.

Castillo, M.M., R. Barba-Álvarez, \& A. Mayorga. 2018. Riqueza y diversidad de insectos acuáticos en la cuenca del río Usumacinta en México. Revista Mexicana de Biodiversidad 89:45-64.

Cedeño-Vásquez, J.R., P. Ross \& S. Calmé. 2006. Population status and distribution of Crocodylus acutus and C. moreletii in southeastern Quintana Roo, Mexico. Herpetological Natural History 10:17-30.
Cedeño-Vásquez, J.R. \& S.D. Pérez-Rivera. 2010. El Cocodrilo de Pantano (Crocodylus moreletii) en Laguna Esmeralda, Quintana Roo, México. Revista Latinoamericana de Conservación 2:91-98.

Cedeño-Vázquez, J. 2016. Crocodylus moreletii. Cannibalism. Mesoamerican Herpetology 3:470-472.

CONAP (Consejo Nacional de Áreas Protegidas). 2008. Plan Maestro 2008-2012. Áreas Protegidas del Suroeste de Petén. (Editado y revisado por Claudia Santizo, Erick Ponciano, Estuardo Secaira, Rudy Herrera, Sergio Guzmán, Gabriel Alonso, Bertila Bailey y Salvador López). Unidad Ejecutora de Coordinación del Programa para el Desarrollo Sostenbile de Petén/ Centro Agronómico Tropical de Investigación y Enseñanza - Ministerio de Agricultura Ganadería y Alimentación/ Banco Interamericano de Desarrollo (UEC PDS/ CATIE-MAGA/BID). Guatemala.

CONAP (Consejo Nacional de Áreas Protegidas). 2015. Plan Maestro de la Reserva de la Biósfera Maya. Segunda Actualización. Tomo 1. Documento Técnico No. 20-2016. Guatemala.

CONAP-DGPCN/MICUDE (Consejo Nacional de Áreas Protegidas Dirección General del Patrimonio Cultural y Natural/ Ministerio de Cultura y Deportes). 2015. Plan Maestro del Parque Nacional Yaxha, Nakum, Naranjo. Primera Actualización. (Editado y revisado por Carlos Rodríguez Olivet, Julio Rafael Morales, Oscar Quintana, Jenniffer Ortiz, Julio López Payés). CATIE-GITEC Consult GmbH y Programa para el Desarrollo de Petén para la Conservación de la Reserva de la Biosfera Maya (PDPCRBM/ MARN). Guatemala.

CITES (Convención sobre el Comercio Internacional de Especies Amenazadas de Fauna y Flora Silvestres). 2016. Examen De Las Propuestas De Enmienda A Los Apéndices I y II, Información complementaria sobre el estado de las poblaciones silvestres y manejo de cocodrilo de pantano Crocodylus moreletii en México. Johannesburgo, Sudáfrica.

Cruz-Paz, G., M.M., Castillo, A. Espinoza-Tenorio, L.C. Bravo-Peña, E. Valencia Barrera \& M.A. Mesa-Jurado. 2018. Áreas prioritarias de conservación en la cuenca Usumacinta. La aplicación de un enfoque multicriterio. Investigaciones Geográficas. UNAM. No. 97.

Dever, J., R., Strauss, T. Rainwater, S.T. McMurry \& L. Densmore. 2002. Genetic Diversity, Population Subdivision, and Gene Flow in Morelet's Crocodile (Crocodylus moreletii) from Belize, Central America. Copeia Review: Genetics and Evolution 4:1078-1091. 
Escobedo-Galván, A.H. 2008. Estructura poblacional y proporción de sexos en Caiman crocodilus en Caño Negro, Costa Rica. Iheringia. Série Zoología 98:489-492.

Escobedo-Galván, A.H., G. Casas-Andreu, G. Barrios-Quiroz, V. H. Sustaita-Rodríguez \& M.A. López Luna. 2011. Observations on nests of Crocodylus moreletii in San Luis Potosí, Mexico. Revista Mexicana de Biodiversidad 82:315-317.

González-Espinosa, M., N. Ramírez-Marcial \& L. Ruiz-Montoya. 2005. Diversidad Biológica en Chiapas. México, D.F.

Hammer, Ø., D.A.T. Harper \& P.D. Ryan. 2013. PAST: Paleontological Statistics software package for education and data analysis. Palaeontologia Electronica 4:1-9.

Köhler, G. 2003. Reptiles of Central America. Herpeton, Germany.

Lara, Ó. 1990. Estimación del tamaño y estructura de la población de Crocodylus moreletii en los lagos Petén Itzá, Sal-Petén, Petenchel y Yaxhá, El Petén, Guatemala. Tesis de Maestría, Universidad Nacional, Heredia. Costa Rica.

March, I. \& M. Castro. 2010. La Cuenca del Usumacinta: Perfil y perspectivas para su conservación y desarrollo sustentable. Pp. 193-197. En C.A.H. (Eds.). Las cuencas hidrográficas de México. Diagnóstico y priorización. Universidad de San Luís Potosí. México.

Mendoza-Carranza, M., W. Arévalo-Frías, A. Espinoza-Tenorio, C.C. Hernández-Lazo, A. Álvarez-Merino, \& R. Rodiles-Hernández. 2018. La importancia y diversidad de los recursos pesqueros del río Usumacinta, México. Revista mexicana de biodiversidad 89:131-146.

Palmer, M.L. \& F.J. Mazzotti. 2004. Structure of everglades alligator holes. Wetlands 24:115-122.

Platt, S. G. \& J.B. Thorbjarnarson. 2000. Population status and conservation of Morelet's crocodile, Crocodylus moreletii, in northern Belize. Biological Conservation 96:21-29.

Platt, S.G., T.R. Rainwater, A. G. Finger, J. B. Thorbjarnarson, T. A. Anderson \& S. T. Mcmurry. 2006. Food habits, ontogenetic dietary partitioning and observations of foraging behaviour of Morelet's crocodile (Crocodylus moreletii) in northern Belize. Herpetological Journal 16:281-290.
Read, M.A., D. Miller Jeffrey, I.P. Bell \& A. Felton. 2004. The distribution and abundance of the estuarine crocodile, Crocodylus porosus, in Queensland. Wildlife Research 31:527-534.

Reyes Morales, E.M., J.E. Morales Can, B.E. Oliva Hernández \& C.V. Dávila Pérez. 2009. Los Cuerpos de Agua de la Región Maya Tikal -Yaxhá: Importancia de la Vegetación Acuática Asociada, Calidad de Agua y Conservación. Programa Universitario de Investigación en Recursos Naturales y Ambiente -PIURNADirección General de Investigación. Universidad San Carlos de Guatemala.

Rodas-Trejo, J., P. Ocampo-González, H. Mandujano Camacho, R. Grajales Zepeda \& J. Hernández-Nava. 2017. Estado poblacional del Crocodylus moreletii (Reptilia: Crocodylidae) en el Área Natural Protegida Laguna de Términos, Campeche, México Population status of Crocodylus moreletii (Reptilia: Crocodylidae) in the Laguna de Términos protected natural area, Campeche, Mexico. Quehacer Científico en Chiapas 12:41-46.

Ross, C.A. 1987. Crocodylus moreletii. Catalog of American Amphibians and Reptiles 407:1-407.

Rueda Cordero, B.A., M.A. López Luna \& L.D. Olivera Gómez. 2017. Uso de hábitat del cocodrilo de pantano Crocodylus moreletii en una laguna urbanizada en México. Quehacer Científico en Chiapas 12:35-40.

Sánchez Herrera, O.G., López Segurajáuregui, A. García Naranjo Ortiz de la Huerta \& H. Benítez Díaz. 2011. Programa de Monitoreo del Cocodrilo de Pantano (Crocodylus moreletii) MéxicoBelice-Guatemala. Comisión Nacional para el Conocimiento y Uso de la Biodiversidad. México.

Somaweera, R., J. Nifong, A. Rosenblatt, M.L. Brien, X. Combrink, R.M. Elsey, G. Grigg, W. E. Magnusson, F.J. Mazzoti, A. Pearcy, S.G. Platt, M.H. Shirley, M. Tellez, J. V.D. Ploeg, G. Webb, R. Whitaker \& B.L. Webber. 2020. The ecological importance of crocodylians: towards evidence-based justification for their conservation. Biological Reviews 95:936-959.

Tellez, M., M. Boucher, \& K. Kohlman. 2016. Population status of the American Crocodile (Crocodylus acutus) in Caye Caulker, Belize. Mesoamerican Herpetology 3:450-460.

Tellez, M., B. Arévalo, I. Paquet-Durand \& S. Heflick. 2017. Population status of Morelet's Crocodile (Crocodylus moreletii) in Chiquibul Forest, Belize. Mesoamerican Herpetology 4:8-2 
Thorbjarnarson, J.B. 2010. American Crocodile Crocodylus acutus. Pp. 46-53. In S.C. Manolis \& C. Stevenson (Eds). Crocodiles. Status Survey and Conservation Action Plan. Third Edition. Crocodile Specialist Group: Darwin.

Villalobos-Zapata, G.J., \& J. Mendoza Vega (Coord.). 2010. La Biodiversidad en Campeche: Estudio de Estado. Comisión Nacional para el Conocimiento y Uso de la Biodiversidad (CONABIO), Gobierno del Estado de Campeche, Universidad
Autónoma de Campeche, El Colegio de la Frontera Sur. México.

Villegas, A., \& V. Reynoso. 2013. Relative abundance and habitat preference in isolated populations of Morelet's crocodile (Crocodylus moreletii) along the coast of the Gulf of Mexico. Herpetological Conservation and Biology 8:571-580. 